\title{
IntSplice: prediction of the splicing consequences of intronic single-nucleotide variations in the human genome
}

\author{
Akihide Shibata $^{1}$, Tatsuya Okuno ${ }^{1}$, Mohammad Alinoor Rahman ${ }^{1}$, Yoshiteru Azuma ${ }^{1}$, Jun-ichi Takeda ${ }^{1}$, \\ Akio Masuda ${ }^{1}$, Duygu Selcen ${ }^{2}$, Andrew G Engel ${ }^{2}$ and Kinji Ohno ${ }^{1}$
}

Precise spatiotemporal regulation of splicing is mediated by splicing cis-elements on pre-mRNA. Single-nucleotide variations (SNVs) affecting intronic cis-elements possibly compromise splicing, but no efficient tool has been available to identify them. Following an effect-size analysis of each intronic nucleotide on annotated alternative splicing, we extracted 105 parameters that could affect the strength of the splicing signals. However, we could not generate reliable support vector regression models to predict the percent-splice-in (PSI) scores for normal human tissues. Next, we generated support vector machine (SVM) models using 110 parameters to directly differentiate pathogenic SNVs in the Human Gene Mutation Database and normal SNVs in the dbSNP database, and we obtained models with a sensitivity of $0.800 \pm 0.041$ (mean and s.d.) and a specificity of $0.849 \pm 0.021$. Our IntSplice models were more discriminating than SVM models that we generated with Shapiro-Senapathy score and MaxEntScan::score3ss. We applied IntSplice to a naturally occurring and nine artificial intronic mutations in RAPSN causing congenital myasthenic syndrome. IntSplice correctly predicted the splicing consequences for nine of the ten mutants. We created a web service program, IntSplice (http://www.med.nagoya-u.ac.jp/neurogenetics/IntSplice) to predict splicing-affecting SNVs at intronic positions from -50 to -3 . Journal of Human Genetics (2016) 61, 633-640; doi:10.1038/jhg.2016.23; published online 24 March 2016

\section{INTRODUCTION}

Higher eukaryotes have evolved by acquiring tissue-specific and developmental stage-specific regulation of alternative splicing of pre-mRNA rather than by acquiring novel genes. ${ }^{1}$ Precisely regulated splicing process takes place in the spliceosome, which comprises five small nuclear ribonucleoproteins (U1, U2, U4, U5 and U6 snRNPs) and a large number of non-snRNP proteins. ${ }^{2}$ In the first step of the assembly of the spliceosome, U1 snRNP, SF1, U2AF65 and U2AF35 bind to the splicing cis-elements at the $5^{\prime}$ splice site (ss), the branch point sequence (BPS), the polypyrimidine tract (PPT), and the $3^{\prime} \mathrm{ss}$, respectively. ${ }^{3,4}$ Single-nucleotide variations ( $\mathrm{SNVs}$ ) disrupting these essential cis-elements lead to aberrant splicing and cause human diseases. At least $10 \%$ of inherited human diseases are caused by mutations affecting the essential splicing cis-elements at the $5^{\prime}$ and $3^{\prime}$ ss's. ${ }^{5}$ In addition, intronic and exonic splicing cis-elements also confer precise spatiotemporal regulation of constitutive and alternative splicing, which are also frequently disrupted in human diseases. ${ }^{6}$ Development of high-throughput sequencing technologies has enabled us to obtain a large number of SNVs from a significant number of individuals. Prediction of the splicing consequences of intronic SNVs, however, remains difficult due to the lack of efficient prediction tools.
Exonic SNVs often disrupt or de novo generate exonic splicing enhancers and silencers. Several exonic splicing enhancer/exonic splicing silencer search tools are available online: exonic splicing enhancer finder $3.0,{ }^{7}$ ESRsearch,${ }^{8}$ FAS-ESS, ${ }^{9}$ PESXs, ${ }^{10,11}$ RESCUE-ESE, ${ }^{12}$ Human Splicing Finder, ${ }^{13}$ SpliceAid, ${ }^{14}$ SpliceAid2, ${ }^{15}$ CRYP-SKIP, ${ }^{16}$ Spliceman ${ }^{17}$ and RegRNA 2.0. ${ }^{18}$ These tools can be used to predict splicing consequences of exonic SNVs. In contrast to a variety of available tools for inspecting exonic SNVs, only two tools are available to our knowledge to score the $3^{\prime}$ ss. The ShapiroSenapathy score is calculated using the position-specific scoring matrix, representing the frequency of each nucleotide from intronic position -14 (Int-14) to exonic position $+1(E x+1),{ }^{19}$ which has long been used to predict the splicing effects of SNVs. The MaxEntScan:: score 3 ss scores the $3^{\prime}$ ss from Int-20 to Ex+3. ${ }^{20}$ Shapiro-Senapathy score and MaxEntScan, however, were not specifically designed to predict the splicing consequences of intronic SNVs.

We have previously reported that the consensus sequence of human BPS is yUnAy, where ' $y$ ' represents pyrimidines and ' $n$ ' represents any nucleotides. ${ }^{21}$ Similarly, extensive analyses of human branch points using RNA-seq show that the consensus BPS sequence is ' $U n A y$ '. ${ }^{22-24}$ The highly degenerative BPS motif, however, prevented us from

\footnotetext{
${ }^{1}$ Division of Neurogenetics, Center for Neurological Diseases and Cancer, Nagoya University Graduate School of Medicine, Nagoya, Japan and ${ }^{2}$ Department of Neurology, Mayo Clinic, Rochester, MN, USA

Correspondence: Professor K Ohno, Division of Neurogenetics, Center for Neurological Diseases and Cancer, Nagoya University Graduate School of Medicine, 65 Tsurumai, Showa-ku, Nagoya 466-8550, Japan.

E-mail: ohnok@med.nagoya-u.ac.jp

Received 19 June 2015; revised 23 February 2016; accepted 24 February 2016; published online 24 March 2016
} 
developing a model to predict the position and the splicing effect of BPS. We also reported that a mutation at the first nucleotide of an exon causes aberrant splicing at the AG-dependent $3^{\prime}$ ss, where a short PPT cannot confer sufficient binding affinity for U2AF65 and additional binding of U2AF35 to the $3^{\prime}$ ss is required. ${ }^{25}$ Here, we present a support vector machine (SVM) model, IntSplice, to predict aberrant splicing because of intronic SNVs (Int-SNVs) at positions from Int-50 to Int-3 (Int-50:Int-3).

\section{MATERIALS AND METHODS}

\section{Ethics statement}

Studies on a patient with congenital myasthenic syndrome were approved by the ethical review committees of the Mayo Clinic and the Nagoya University Graduate School of Medicine. The studies were performed after an appropriate informed written consent was obtained.

\section{Databases}

Sequence motifs of the splicing trans-factors were obtained from the SpliceAid database. ${ }^{14}$ Exonic and intronic positions of these sequence motifs were not taken into account, because: (i) Int-SNVs should not change any exonic motifs; (ii) we did not look into exonic nucleotides when we made our models; and (iii) exonic and intronic positions were not always available in the SpliceAid database. RNA-seq data on the brain, cerebral cortex, heart, liver, skeletal muscle and lungs in normal humans were obtained from the GEO database (the accession number, GSE13652) in an SRA format. ${ }^{26}$ The number of individuals and their demographic features for each tissue were not available for GSE13652. ${ }^{26}$ RNA-seq data on the breasts, lymph nodes, testes, adipose tissue, colon, skeletal muscle, liver and brain in normal humans were similarly obtained with the GEO accession number GSE12946 in an SRA format. ${ }^{27}$ For GSE12946, each tissue sample was obtained from a single unrelated individual. ${ }^{27}$ The SRA files were converted to fastq files using an SRA toolkit (http://eutils.ncbi.nih.gov/Traces/sra/sra.cgi?view = software). Disease-causing mutations located at positions Int-50:Int-3 were obtained from the Human Gene Mutation Database (HGMD) Professional (Biobase, Wolfenbüttel, Germany). Some intronic mutations in the HGMD might not be splicing mutations and might affect a transcription enhancer/silencer, a pre-miRNA sequence, or a yet uncharacterized cis-element, but the functional consequences of intronic mutations were not always deeply dissected in original papers. We therefore included all intronic mutations at positions Int-50:Int-3, without filtering out non-splicing mutations. Normal SNVs were obtained from dbSNP134. SNVs included in the HGMD were excluded from our analysis. We also excluded SNVs with a global minor allelic frequency of $<0.01$.

\section{Support vector regression and support vector machine modeling}

The RNA-seq data were mapped to the human genome GRCh37/hg19 with ENSEMBL release 64 using TopHat mapper with its default parameters. ${ }^{28}$ Splicing efficiency of each individual exon (percent-spliced-in score, PSI) was calculated with the MISO software..$^{29}$ For each RNA-seq data set of the 14 human tissues, we randomly divided 3' ss's into five groups. Four groups were arbitrarily chosen to generate an SVR model with the nu-SVR functionality of LIBSVM version $3.17^{30}$ to predict PSIs using 105 parameters. We then tested the validity of the generated SVR model using the remaining fifth group. We made five SVR models by changing the training and validation groups. We generated 100 different combinations of five groups for each RNA-seq data set and ran the SVR modeling 500 times.

SVM models to distinguish between pathogenic and normal Int-SNVs were generated with 110 parameters using the C-SVC functionality of LIBSVM. ${ }^{30}$ A total of 500 different SVM models were generated for 100 different data sets of 1162 pathogenic and 1162 normal Int-SNVs. Normal Int-SNVs in each data set were randomly selected from 16741 normal SNVs. For SVM modeling, we compared four kernels of 'linear', 'polynomial', 'radial basis function' and 'sigmoid'.

For both the SVR and the SVM models, scores of each parameter were normalized using the SVM-scale functionality of LIBSVM, ${ }^{30}$ so that each parameter was equally weighted. Perl scripts were run on the RPIMERGY CX400 UNIX server (Fujitsu, Kawasaki, Japan).

\section{A patient with congenital myasthenic syndrome}

The patient, now 29 years old, was hypomotile in utero. After birth, he was floppy, had a poor cry, needed ventilatory support and had arm and leg contractures. He improved gradually and walked at the age of 14 months. He showed a decremental electromyographic response to repetitive nerve stimulation in several muscles. His weakness was improved with a cholinesterase inhibitor, pyridostigmine. Sanger sequencing of genomic DNA revealed a homozygous T-to-A substitution at intron 5 (c.913-5T > A) of the RAPSN gene. No muscle specimen was available from the patient.

\section{Minigene constructs}

To construct the human RAPSN minigene, we amplified a genomic segment spanning exons 5-7 of RAPSN by PCR with KOD Plus DNA polymerase (Toyobo, Osaka, Japan) using genomic DNA isolated from HeLa cells. The 5' ends of the forward and reverse primers carried the BamHI and XhoI sites, respectively. The amplified fragment was cloned into the BamHI and XhoI sites of the pcDNA3.1(+) vector (Invitrogen, Carlsbad, CA, USA) to generate the pcDNA-RAPSN minigene. The naturally occurring (patient) and artificial mutations were engineered into the pcDNA-RAPSN construct using the QuikChange Site-Directed Mutagenesis Kit (Stratagene, San Diego, CA, USA). The presence of artifacts was excluded by sequencing the entire inserts.

\section{Cell culture, transfection, and PCR with reverse transcription for splicing analysis}

HeLa cells were cultured in DMEM (Sigma-Aldrich, St Louis, MO, USA) with $10 \%$ fetal bovine serum (FBS, Sigma-Aldrich). The cells were plated $24 \mathrm{~h}$ before transfection in six-well culture plates $\left(1.5 \times 10^{5}\right.$ cells per well), and transfected using the FuGENE 6 transfection reagent (Roche, Basel, Switzerland) according to the manufacturer's instructions. Total RNA was extracted $40 \mathrm{~h}$ following transfection using the TRIzol reagent (Invitrogen), followed by DNase I treatment. The cDNA was synthesized with an oligo-dT primer using the ReverTra Ace reverse transcriptase (Toyobo). PCR-amplification was performed using the GoTaq DNA polymerase (Promega, Madison, WI, USA) with the following primer pair: $5^{\prime}$-ATCATGACCGAGATCGGAAAC- $3^{\prime}$ on exon 5 and 5'-GTGGAACCTCACAACGTGC-3' on exon 7.

\section{MS2-affinity purification of a spliceosomal complex}

To synthesize an RNA substrate for the MS2-affinity purification of a spliceosomal complex, we first amplified a genomic segment spanning RAPSN exons 5 and 6 from wild-type and mutant pcDNA-RAPSN minigenes, and then cloned them into the BamHI and XhoI sites of pcDNA3.1(+) to generate the pcDNA-RAPSN-E5-E6 minigenes. A segment spanning three copies of the MS2-binding sites was PCR-amplified from pSP64-MS2 that we previously reported, ${ }^{31}$ and was introduced downstream of exon 6 of the pcDNA-RAPSN-E5-E6 minigenes using the megaprimer method. ${ }^{32}$ The generated pcDNA-RAPSN-E5-E6-MS2 minigenes were used as templates to synthesize RNA-substrates using the RiboMAX System (Promega).

An RNA probe (1 pmol) was incubated with 20-fold molar excess of the MS2-MBP fusion protein. ${ }^{33}$ Fifty microliters of HeLa nuclear extract (CilBiotech, Mon, Belgium) was preincubated with $10 \mu$ (bead volume) of amylose resin (New England Biolabs, Ipswich, MA, USA) overnight at $4{ }^{\circ} \mathrm{C}$. The purified HeLa nuclear extract was then incubated at $37^{\circ} \mathrm{C}$ for $30 \mathrm{~min}$, with a mixture of the RNA probe and the MS2-MBP fusion protein at final concentrations of $60 \mathrm{~mm} \mathrm{KCl}$ and $25 \%$ HeLa nuclear extract. Ten microliters (bead volume) of amylose resin was added and mixed on a rotary shaker at $4{ }^{\circ} \mathrm{C}$ for $30 \mathrm{~min}$. After washing four times with washing buffer (20 mM HEPES at $\mathrm{pH}$ $8.0,150 \mathrm{~mm} \mathrm{KCl}$, and $0.05 \%$ Triton X-100), the resin-bound molecules were eluted with $10 \mathrm{~mm}$ maltose solution. The purified proteins were subjected to SDS-polyacrylamide gel electrophoresis and immunoblot analyses to detect the binding of U2AF65, U2AF35 and U1 snRNP (U1-70 K), respectively. The antibodies used were U2AF65 (MC3, sc-53942, Santa Cruz Biotechnology, 


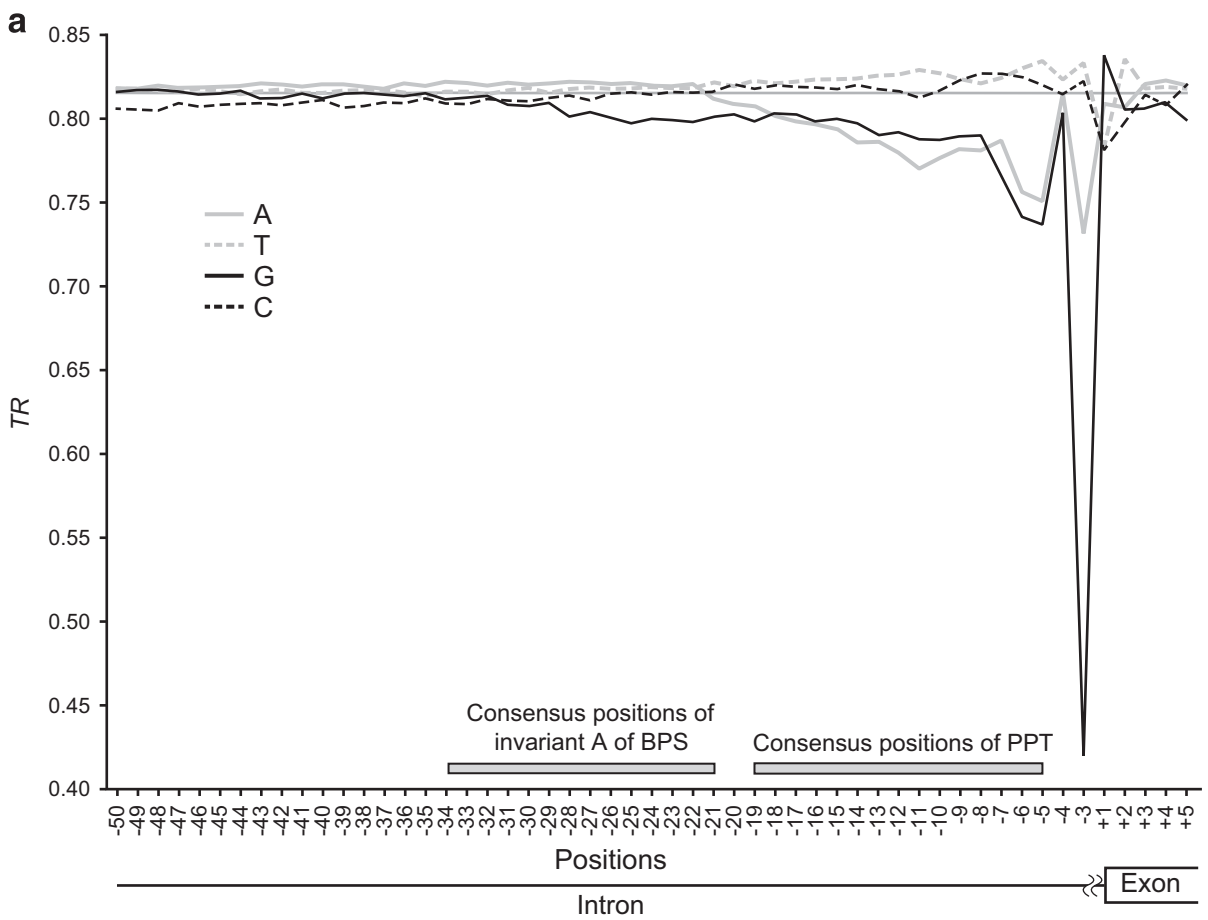

b

yUnAynnYYYYYYYYYYYYYYYYYYYYY - YAG G
$\begin{aligned} & \text { Polypyrimidine tract at } \\ & 4 \text { to } 24 \text { nt. downstream } \\ & \text { of invariant A of BPS }\end{aligned}$
$\begin{aligned} & 21-34 \text { nt. upstream } \\ & \text { of the 3' end of intron }\end{aligned}$

Figure 1 Annotation-based analysis of the effects of intronic nucleotides on splicing. (a) The effect of each intronic nucleotide at positions Int-50:Int-3 and $E x+1: E x+5$ on the average TR (see Supplementary Figure 2) according to the ENSEMBL annotation 64 . For example, G at position Int-3 is frequently observed in alternatively spliced 3' Ss, yielding a markedly reduced TR. (b) Schematic representation of the consensus nucleotide compositions of the BPS (arrow) and PPT.21

Dallas, TX, USA), U2AF35 (N-16, sc-19961, Santa Cruz Biotechnology), and U1-70 K (H111, kindly provided by Dr Akila Mayeda at the Fujita Health University).

\section{RESULTS}

Estimation of the effects of individual intronic nucleotides on splicing annotated in the ENSEMBL release 64 database

A diagram showing the flow of our analyses in this communication is shown in Supplementary Figure 1. We first inspected the alternative splicing events annotated in the ENSEMBL release 64 on the GRCh37/hg19 human genome. We restricted our analysis to introns with 'AG' dinucleotides at the 3 ' end, and not 'AC'. We estimated the splicing efficiency of the 3 ' ss by defining a new parameter, the transcription ratio $(T R)$. When a gene gives rise to $m$ different transcripts at a specific position, and $n$ transcripts are spliced at the $3^{\prime}$ ss according to ENSEMBL release 64, we defined TR for that specific $3^{\prime}$ ss as $n / m$. An example of TRs is shown in Supplementary Figure 2. Assuming that the $3^{\prime}$ ss with a high TR carries a strong splicing signal, we plotted the average $T R$ against individual nucleotides from positions Int-50 to $\mathrm{Ex}+5$ at the $3^{\prime}$ ss. The plot revealed that nucleotides at positions Int-13:Int-5, Int- $3, \mathrm{Ex}+1$ and $\mathrm{Ex}+2$ were critical determinants of TR (Figure 1).

\section{Prediction of PSIs of 14 tissue-specific RNA-seq data using SVR modeling}

The PSIs of individual $3^{\prime}$ ss's in the RNA-seq data of 14 normal human tissues in GSE13652 $2^{26}$ and GSE12946 ${ }^{27}$ were calculated with MISO. ${ }^{29}$ We first tried to predict a PSI using the primary nucleotide sequence with a linear regression model. If we can efficiently predict the PSI of a given $3^{\prime}$ ss, we should be able to make a model to identify an intronic splicing mutation. The primary nucleotide sequence alone at positions Int-50:Ex +5 , however, was not sufficient to predict the PSI of a given $3^{\prime}$ ss (data not shown). We then predicted a PSI using an SVR model. We extracted 105 parameters that possibly dictate the strength of the splicing signals (Supplementary Table 1). The 105 parameters included individual nucleotides at positions Int-3 and Ex+1 according to Figure 1, the sequence motifs of all the splicing trans-factors in the SpliceAid database, ${ }^{14}$ the position weight matrix of the human BPS $^{21}$ (Supplementary Table 2), variable definitions of PPT, $\Delta \mathrm{G}$ of a predicted secondary RNA structure based on the mfold program, ${ }^{34}$ and so on. We included $\Delta \mathrm{G}$ of mfold, because the secondary RNA structure is a critical determinant of the splicing consequences. ${ }^{35-37}$ The RNA-seq data of the 14 tissues, however, generated SVR models with correlation coefficients $(R)$ ranging from 0.239 to 0.274 (mean and s.d., $0.253 \pm 0.011$ ) (Supplementary Figure 3 ). These SVR models 
thus failed to predict the PSIs with enough accuracy to estimate the splicing strength of a given $3^{\prime}$ ss, and their sole application could not predict the splicing consequence of a given Int-SNV.

\section{Differentiation of pathogenic and normal Int-SNVs using SVM modeling}

Next, we tried to differentiate pathogenic SNVs registered in the HGMD and normal SNVs in the dbSNP database at positions Int-50: Int-3. In addition to 14 PSIs calculated with the 14 SVR models stated above, we used the 105 parameters once again to make a prediction model. Among the 119 parameters, however, we excluded 10 parameters that represented the nucleotides at positions Int-2:Ex+5 and at the $5^{\prime}$ ss, where no SNV should exist in the current analysis. We also added a parameter indicating whether an 'AG' dinucleotide is generated de novo by Int-SNVs. We thus used a total of 110 parameters (Supplementary Table 1) to make SVM models. The HGMD included 1162 pathogenic SNVs at positions Int-50:Int-3, whereas the dbSNP database included 16741 normal SNVs at positions Int-50:Int-3 with a global minor allelic frequency of $>0.01$. To match the numbers of SNVs in HGMD and the dbSNP database, we randomly chose 1162 SNVs from the 16741 SNVs in the dbSNP database. A data set of 2324 pathogenic and normal SNVs was divided into five groups. The data sets of 2324 SNVs were generated 100 times in order to validate the models repeatedly. For each data set, four groups were employed to generate an SVM model (IntSplice) with LIBSVM $^{30}$ using the 110 parameters to predict whether an SNV belongs to the HGMD or the dbSNP database. We then tested the validity of the SVM model generated using the remaining fifth group, and calculated the sensitivity and the specificity of each model. A total of 500 different SVM models were generated with each of four kernels of 'linear', 'polynomial', 'radial basis function' and 'sigmoid', respectively (Table 1 ). The sensitivity ranged from 0.710 to 0.769 , and the specificity ranged from 0.896 to 0.936 . Among the four kernels, the radial basis function generated the most efficient SVM models.

The three best parameters in SVM modeling were the MaxEnt score at Int-20:Int-3 (coefficient $=-12.7$ ), the Shapiro-Senapathy score at Int-50:Int-3 (coefficient $=-11.2$ ), and the ratio of A/G's at Int-20:Int$8($ coefficient $=10.8)($ Supplementary Table 1$)$. As the MaxEnt score and the Shapiro-Senapathy score are comprehensive parameters to dictate the strength of splicing signals, these two parameters were better than individual parameters. Among the individual parameters, the coefficient of the ratio of A/G's at Int-20:Int- 8 was as high as those of the comprehensive parameters. A similar and partly overlapping parameter was the number of G's at Int-12:Int-3 (coefficient=7.83). Contribution of these individual parameters in SVM modeling suggests that the presence of purines at PPT has a marked negative effect on splicing.

Inclusion of SVR-based prediction of PSI may bias the SVM models in favor of 14 tissues that were included in the SVR modeling. We thus made 500 SVM models without SVR-based prediction of PSI derived from 14 RNA-seq data, and compared the sensitivity and specificity to those with SVR-based prediction of PSI. We found that the sensitivities and specificities of the two SVM models with and without PSI parameters were essentially the same (Supplementary Table 3). As the sum of specificity and sensitivity at Int-50:Int-3 became marginally low by exclusion of PSI parameters, we included PSI parameters in the following analyses.

We also made SVM models with 1162 pathogenic Int-SNVs and 16741 normal Int-SNVs at positions Int-50:Int-3 using the radial basis function (unmatched models). We generated 500 different data sets by randomly selecting four-fifth of pathogenic/normal Int-SNVs as a training data set and the remaining one-fifth of pathogenic/normal Int-SNVs as a validation data set. SVM models with unmatched data sets had a sensitivity of $0.762 \pm 0.030$ (mean and s.d.) and a specificity of $0.905 \pm 0.024$ (mean and s.d.). As shown in Table 1, SVM models with matched data sets had a sensitivity of $0.899 \pm 0.022$ (mean and s. d.) and a specificity of $0.772 \pm 0.027$ (mean and s.d.). Although the sums of sensitivity and specificity were similar between the two data sets ( 1.671 for unmatched data sets and 1.667 for matched data sets), sensitivity was higher with the matched data sets and specificity was higher with the unmatched data sets. With unmatched data sets, the number of normal SNVs was 14 times $(=16741 / 1162)$ higher than that of pathogenic SNVs. SVM models with unmatched data sets were thus in favor of predicting that Int-SNVs were negative, and specificity became high $(0.905)$ at the cost of low sensitivity (0.762). We supposed that both unmatched and matched models could be used for different purposes. However, in order to detect pathogenic Int-SNVs identified in human diseases, we hoped to keep the sensitivity high as much as possible, and we used SVM models with matched data sets in the following analyses.

Comparison of IntSplice with SVM models generated based on the Shapiro-Senapathy score and MaxEntScan::score3ss

Although Shapiro-Senapathy score ${ }^{19}$ and MaxEntScan::score $3 s^{20}$ are not designed to predict aberrant splicing due to Int-SNVs, we exploited these scores to predict the splicing consequences of Int-SNVs by setting an automatic cutoff value with SVM. For each of the 100 data sets comprising the 2324 Int-SNVs at positions Int-50: Int-3 that we used for the IntSplice modeling, we analyzed all the 2324 Int-SNVs at positions Int-50:Int-3 with Shapiro-Senapathy score and 2064 Int-SNVs at positions Int-20:Int-3 with MaxEntScan. Shapiro-Senapathy score was originally designed to score the $3^{\prime}$ ss up to Int-14, and the scoring matrix was based on the nucleotide sequences available in the year $1987 .{ }^{19}$ We thus made a new scoring matrix covering up to Int-50 by analyzing ENSEMBL release 64 (Supplementary Table 2). MaxEntScan was designed to score 3' ss up to Int-20, and was unable to score Int-SNVs at positions Int-50:Int-21. ${ }^{20}$ We randomly divided the data sets comprised of 2324 and 2064 Int-SNVs into five groups. We made 500 SVM models using either the Shapiro-Senapathy score or the MaxEntScan with each of the four kernels of 'linear', 'polynomial', 'radial basis function' and 'sigmoid' (Table 1), as we did with IntSplice. Again, the radial basis function generated the most efficient models with both the Shapiro-Senapathy score and MaxEntScan. The plots of the sensitivity and the specificity of the radial basis function models generated by IntSplice, Shapiro-Senapathy score and MaxEntScan, respectively, revealed that the sum of the sensitivity and the specificity of IntSplice was higher than those of the Shapiro-Senapathy score and MaxEntScan for the Int-SNVs at positions Int-50:Int-3 (Figure $2 \mathrm{a}$ and Table 1), Int-20:Int-3 (Figure 2b and Table 1) and Int-50:Int-21 (Table 1).

\section{IntSplice: a web service program to predict the pathogenic and normal Int-SNVs using SVM modeling}

The aforementioned analyses of the validation data sets indicate that SVM modeling with the radial basis function was able to distinguish between pathogenic and normal Int-SNVs with a sensitivity of $0.772 \pm 0.027$ (mean and s.d.) and a specificity of $0.101 \pm 0.022$ (Table 1). Thus, we generated a global SVM model by including 2324 SNVs and made a web service program, IntSplice, at http://www. med.nagoya-u.ac.jp/neurogenetics/IntSplice. This program accepts a file in a variant call format (VCF) with multiple SNVs and predicts whether each SNV affects splicing or not. A given SNV is mapped to 
Table 1 Comparison of the SVM kernels

\begin{tabular}{|c|c|c|c|c|}
\hline Positions & Tool & SVM kernel & Specificity & Sensitivity \\
\hline Int-50 to Int-3 & PSSM & Linear & $0.890 \pm 0.020$ & $0.560 \pm 0.029$ \\
\hline$\downarrow$ & $\downarrow$ & Polynomial & $0.971 \pm 0.010$ & $0.394 \pm 0.028$ \\
\hline$\downarrow$ & $\downarrow$ & $\begin{array}{l}\text { Radial basis } \\
\text { function }\end{array}$ & $0.889 \pm 0.020^{a}$ & $0.561 \pm 0.029^{a}$ \\
\hline$\downarrow$ & $\downarrow$ & Sigmoid & $0.700 \pm 0.139$ & $0.587 \pm 0.056$ \\
\hline$\downarrow$ & IntSplice & Linear & $0.934 \pm 0.018$ & $0.715 \pm 0.029$ \\
\hline$\downarrow$ & $\downarrow$ & Polynomial & $0.896 \pm 0.022$ & $0.769 \pm 0.028$ \\
\hline$\downarrow$ & $\downarrow$ & $\begin{array}{l}\text { Radial basis } \\
\text { function }\end{array}$ & $0.899 \pm 0.022^{a}$ & $0.772 \pm 0.027^{a}$ \\
\hline$\downarrow$ & $\downarrow$ & Sigmoid & $0.936 \pm 0.019$ & $0.710 \pm 0.030$ \\
\hline Int-20 to Int-3 & PSSM & Linear & $0.704 \pm 0.052$ & $0.623 \pm 0.033$ \\
\hline$\downarrow$ & $\downarrow$ & Polynomial & $0.833 \pm 0.041$ & $0.544 \pm 0.031$ \\
\hline$\downarrow$ & $\downarrow$ & $\begin{array}{l}\text { Radial basis } \\
\text { function }\end{array}$ & $0.831 \pm 0.044^{a}$ & $0.545 \pm 0.032^{a}$ \\
\hline$\downarrow$ & $\downarrow$ & Sigmoid & $0.703 \pm 0.052$ & $0.624 \pm 0.032$ \\
\hline$\downarrow$ & IntSplice & Linear & $0.909 \pm 0.045$ & $0.756 \pm 0.034$ \\
\hline$\downarrow$ & $\downarrow$ & Polynomial & $0.841 \pm 0.052$ & $0.808 \pm 0.028$ \\
\hline$\downarrow$ & $\downarrow$ & $\begin{array}{l}\text { Radial basis } \\
\text { function }\end{array}$ & $0.821 \pm 0.055^{a}$ & $0.817 \pm 0.030^{a}$ \\
\hline$\downarrow$ & $\downarrow$ & Sigmoid & $0.910 \pm 0.054$ & $0.751 \pm 0.034$ \\
\hline$\downarrow$ & MaxEntScan & Linear & $0.937 \pm 0.017$ & $0.663 \pm 0.031$ \\
\hline$\downarrow$ & $\downarrow$ & Polynomial & $0.539 \pm 0.497$ & $0.471 \pm 0.492$ \\
\hline$\downarrow$ & $\downarrow$ & $\begin{array}{l}\text { Radial basis } \\
\text { function }\end{array}$ & $0.924 \pm 0.019^{a}$ & $0.687 \pm 0.033^{a}$ \\
\hline$\downarrow$ & $\downarrow$ & Sigmoid & $0.567 \pm 0.174$ & $0.551 \pm 0.170$ \\
\hline $\begin{array}{l}\text { Int-50 to Int- } \\
21\end{array}$ & PSSM & Linear & $0.989 \pm 0.008$ & $0.056 \pm 0.039$ \\
\hline$\downarrow$ & $\downarrow$ & Polynomial & $0.998 \pm 0.003$ & $0.009 \pm 0.018$ \\
\hline$\downarrow$ & $\downarrow$ & $\begin{array}{l}\text { Radial basis } \\
\text { function }\end{array}$ & $0.998 \pm 0.004^{a}$ & $0.010 \pm 0.019^{a}$ \\
\hline$\downarrow$ & $\downarrow$ & Sigmoid & $0.989 \pm 0.008$ & $0.056 \pm 0.039$ \\
\hline$\downarrow$ & IntSplice & Linear & $0.950 \pm 0.018$ & $0.347 \pm 0.086$ \\
\hline$\downarrow$ & $\downarrow$ & Polynomial & $0.951 \pm 0.018$ & $0.343 \pm 0.083$ \\
\hline$\downarrow$ & $\downarrow$ & $\begin{array}{l}\text { Radial basis } \\
\text { function }\end{array}$ & $0.949 \pm 0.018^{a}$ & $0.352 \pm 0.086^{a}$ \\
\hline$\downarrow$ & $\downarrow$ & Sigmoid & $0.951 \pm 0.018$ & $0.343 \pm 0.084$ \\
\hline
\end{tabular}

Abbreviations: PSSM, position-specific scoring matrix; SVM, support vector machine. Mean and $s . d$ are indicated. MaxEntScan can be applied to SNVs at positions Int-20 to Int-3. asVM modeling with the radial basis function leads to the most discriminating models on average. The sensitivity and the specificity of the radial basis function-based SVM models at positions Int-50:Int-3 and Int-20:Int-3 are plotted in Figure 2.

all the annotated coding transcripts in ENSEMBL 64, and the program analyzes all the transcripts. If an SNV affects splicing of one or more transcript(s), our program predicts that the SNV is pathogenic and shows the affected ENST transcript numbers. Representative results are shown in Figure 3.

\section{Application of the IntSplice program to intronic mutations of RAPSN}

We applied our IntSplice program to the naturally occurring and artificial mutations in RAPSN encoding rapsyn, which makes a scaffold for the muscle nicotinic acetylcholine receptor at the neuromuscular junction. ${ }^{38,39}$ A homozygous RAPSN c.913-5T > A mutation was identified in a patient with congenital myasthenic syndrome. Introduction of a minigene spanning RAPSN exons 5-7 into HeLa cells showed that the RAPSN c.913-5 T > A mutation caused partial skipping of exon 5 (Figure 4a), and compromised binding of U2AF65 (Figure 4b). To investigate which pyrimidine nucleotide in the PPT is essential for splicing of RAPSN exon 5, we first substituted ' $\mathrm{T}$ ' for ' $\mathrm{A}$ ' at position Int- 9 to make a complete stretch of 10 pyrimidines at positions Int-12:Int-3 ('Opt' in Figure 4c). We then serially introduced a mutant ' $A$ ' from positions Int-11 to Int-3. Introduction of the nine artificial mutants into HeLa cells showed that three mutants at positions Int- 6 , Int- 5 and Int-3 led to skipping of exon 5 (Figure 4c). We also found that the binding of U2AF65 to the Int- 6 and Int-5 mutants was compromised, but not that to the Int-3 mutant (Figure $4 \mathrm{~d}$ ). In contrast, the binding of U2AF35 or U1-70 K was not affected in any mutant.

The IntSplice, the MaxEntScan-based model, and the ShapiroSenapathy score-based model correctly predicted aberrant splicing in the patient's mutation, RAPSN c.913-5T $>$ A (Figure 4a). Next, we made the 'Opt' construct as a normal reference sequence, and applied these three models to the nine artificial mutants (Figure 4c). The IntSplice, the MaxEntScan-based model, and the Shapiro-Senapathy score-based model erroneously predicted the splicing consequences in one, two and five mutants, respectively (asterisks in Figure 4c).

\section{DISCUSSION}

In an effort to make a model to predict splicing consequences of Int-SNVs, we first analyzed the position-specific effects of the intronic nucleotides on splicing (Figure 1), and extracted parameters that possibly affect the splicing strength (Supplementary Table 1). We calculated the PSIs of 14 RNA-seq data of normal human tissues, and then tried to predict PSIs using SVR models with the 105 extracted parameters. However, the correlation coefficients between the calculated and predicted PSIs were $<0.3$ (Supplementary Figure 3). Next, we generated SVM models to directly differentiate pathogenic SNVs in the HGMD and normal SNVs in the dbSNP database, with 1 -specificity (a false positive rate) of $\sim 0.10$ and a sensitivity (a true positive rate) of $\sim 0.77$, and named it IntSplice. Inefficient prediction with the RNA-seq-based SVR models suggests that prediction of PSI scores is much more difficult than differentiation between normal and pathogenic Int-SNVs. Although SVM models to differentiate normal and pathogenic Int-SNVs with SVM were better than SVR models to predict PSIs, accurate prediction of splicing consequences of Int-SNVs was not available even with SVM modeling. This was likely due to inadequacy of the training data sets and also to lack of parameters that were essential for splicing regulation in living cells. First, our training data set was comprised of pathogenic Int-SNVs causing Mendelian disorders (HGMD) and normal Int-SNVs in dbSNP with a minor allelic frequency $>0.01$. Neither HGMD nor dbSNP database was comprehensive, and the effects of Int-SNVs that were not present in HGMD or dbSNP could not be estimated. Second, among various parameters that enable precise spatiotemporal regulation of splicing in vivo, the following parameters could not be taken into account in our SVM modeling: (i) splicing is coupled to transcription, which is regulated by RNA polymerase II, other transcription factors, and chromatin structure; ${ }^{40}$ (ii) splicing cis-elements that are functional in specific tissue(s) at specific developmental stage(s) have not been fully characterized; ${ }^{41}$ (iii) the exact mechanisms underlying recognition of degenerative cis-elements by a specific RNA-biding protein remain to be elucidated; ${ }^{42}$ (iv) RNA editing has a pivotal role in spicing, but RNA editing has not been comprehensively characterized; and ${ }^{43}$ (v) Spatiotemporal regulations of expression and activation of splicing trans-factors (RNA-binding proteins) have not been extensively identified. ${ }^{41}$

We compared the prediction efficiency of IntSplice with those of Shapiro-Senapathy score- and MaxEntScan-based SVM models that we generated by applying the same training and validation data sets that were used for IntSplice. Although the sensitivity as well as the sum 
a

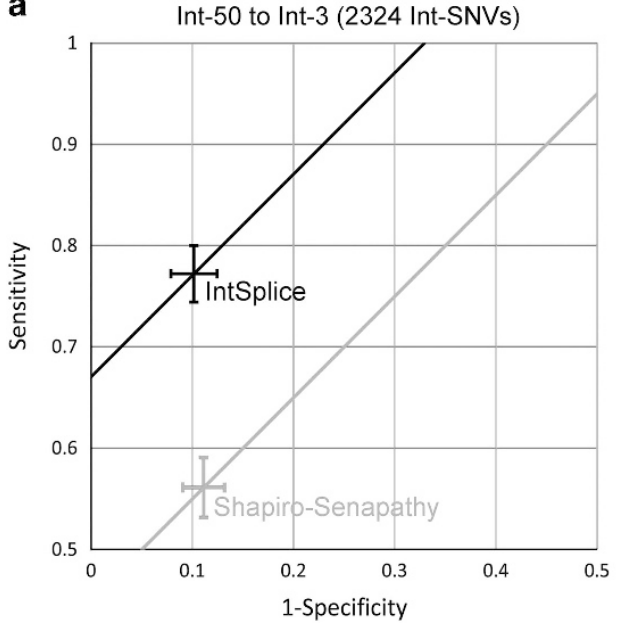

b

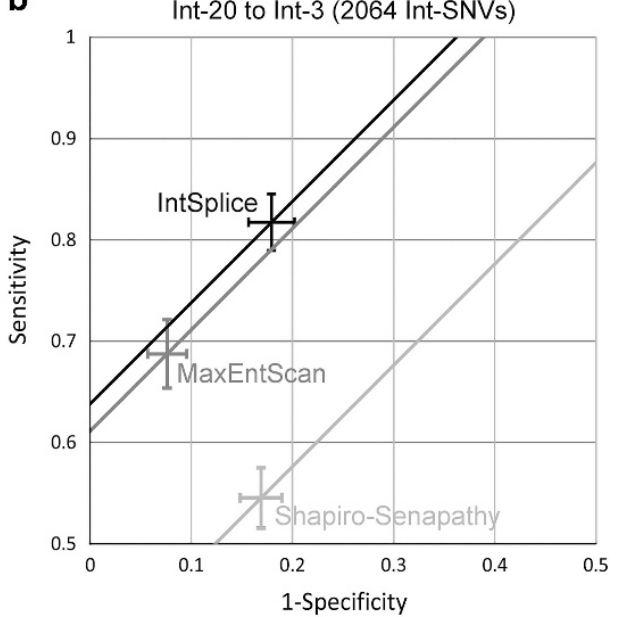

Figure 2 Sensitivities and specificities of IntSplice, the Shapiro-Senapathy score-based model and the MaxEntScan-based model. (a) An SVM model generated by four-fifths of the 2324 normal and pathogenic Int-SNVs in the HGMD and dbSNP databases is applied to the remaining one-fifth of the Int-SNVs. The models are generated five times for 100 different data sets. Bars indicate mean and s.d. As MaxEntScan is unable to score positions Int-50: Int-21, the MaxEntScan-based models in this region are not indicated. (b) IntSplice, the Shapiro-Senapathy score-based model, and the MaxEntScan-based model are generated with 2064 normal and pathogenic Int-SNVs at positions Int-20:Int-3. Mean and s.d. of the sensitivity and the specificity of 500 SVM models are plotted. Oblique lines indicate where the sums of the sensitivity and the specificity are identical. Note that the oblique lines are not receiver operating characteristic (ROC) curves, and are auxiliary lines for comparing the sensitivity and the specificity of three models.

\section{IntSplice Top About Contact}

Column NOTE indicates which exon in which ENSEMBL transcript is predicted to be normal or abnormal.

Columns CHROM to FILTER are included in the uploaded VCF file and are not edited.

\begin{tabular}{|c|c|c|c|c|c|c|c|c|c|}
\hline Af Home & CHROM & POS & ID & REF & ALT & QUAL & FILTER & RESULT & NOTE \\
\hline & chr19 & 11221289 & $\mathrm{dbSNP}$ & C & $T$ & . & . & Normal & $\begin{array}{l}\text { ENSTO00000252444 } \\
\text { _exon7;ENSTO0000 } \\
545707 \text { Exon6;ENS } \\
\text { TO0000535915_exo } \\
\text { n6;ENSTO00004557 } \\
\text { 27_exon5 }\end{array}$ \\
\hline & chr2 & 33359852 & $\mathrm{dbSNP}$ & $\mathrm{G}$ & $\mathrm{T}$ & . & . & Normal & $\begin{array}{l}\text { ENST00000404816 } \\
\text { _exon5;ENST00000 } \\
354476 \text { exon5;ENS } \\
\text { T00000432635_exo } \\
\text { n2 }\end{array}$ \\
\hline & chr10 & 73550880 & HGMD & $\mathrm{G}$ & A & . & . & Abnormal & $\begin{array}{l}\text { ENSTO0000398860 } \\
\text { _exon45;ENST0000 } \\
\text { 0398855_exon45;E } \\
\text { NSTO0000224721_ } \\
\text { exon46 }\end{array}$ \\
\hline & chr17 & 48271548 & HGMD & $\mathrm{G}$ & $\mathrm{T}$ & . & . & Normal & $\begin{array}{l}\text { ENSTO00000225964 } \\
\text { _exon24;ENSTO000 } \\
\text { 0463440_exon2 }\end{array}$ \\
\hline
\end{tabular}

Figure 3 Representative results of the IntSplice web service program (http://www.med.nagoya-u.ac.jp/neurogenetics/IntSplice). Predicted results are shown in the 'RESULT' column. The rightmost 'NOTE' column indicates which exon in which ENSEMBL transcript is predicted to lead to abnormal or normal splicing. The information from the columns 'CHROM' to 'FILTER' is included in the submitted VCF file, and is not edited by IntSplice. For example, a G-to-A transition at position 73550880 of chromosome 10, which is registered in HGMD, is predicted to cause aberrant splicing.

of the sensitivity and the specificity of IntSplice were better than those of Shapiro-Senapathy score-based and MaxEntScan-based models, the specificity of IntSplice was not as good as that of MaxEntScan-based model for Int-SNVs at positions Int-20:Int-3 (Table 1). A high specificity of MaxEntScan was indeed observed in RAPSN mutants. In contrast to IntSplice and Shapiro-Senapathy score-baesd model, MaxEntScan-based model erroneously predicted that the Int-6 and Int-5 mutants were normally spliced, although these caused exon 
a
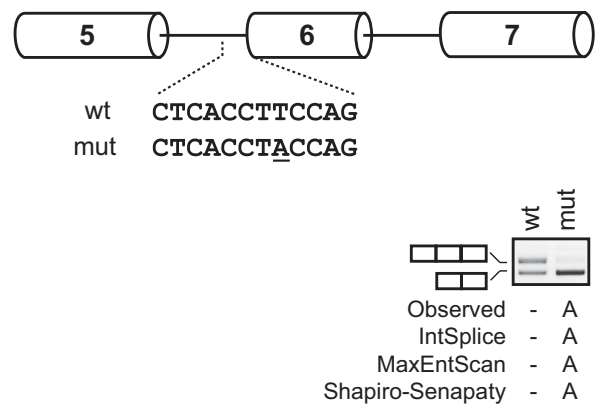

C
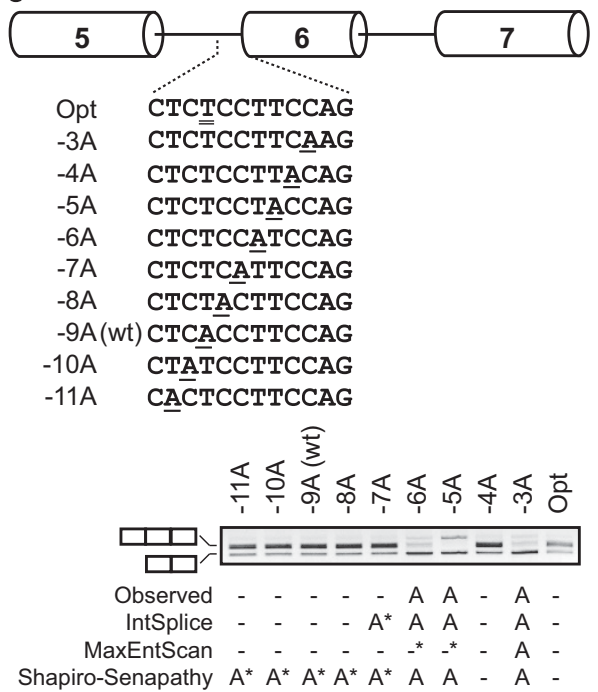

b
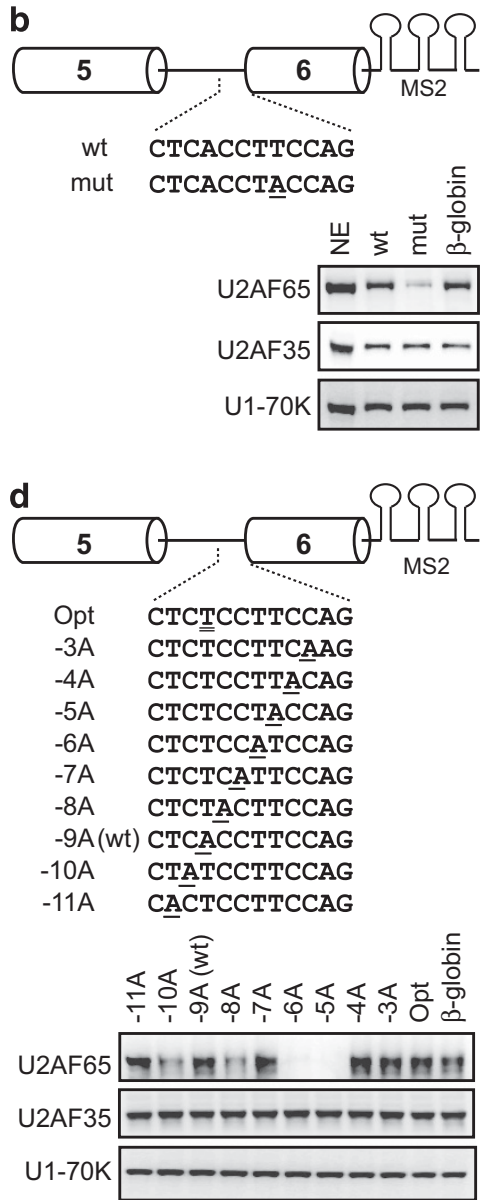

Figure 4 Characterization of the RAPSN c.913-5T>A mutation identified in a patient with congenital myasthenic syndrome and of nine artificial mutations. (a) Schematic representation of pcDNA-RAPSN minigene harboring wild-type (wt) and mutant c.913-5T > A (mut) sequences. PCR with reverse transcription of the minigenes introduced into HeLa cells are shown. 'A', aberrant splicing; '-', normal splicing. IntSplice, the Shapiro-Senapathy score-based model, and the MaxEntScan-based model correctly predict aberrant splicing. (b) Schematic representation of MS2-attached wild-type (wt) and mutant (mut) RNA substrates that originated from pcDNA-RAPSN-E5-E6-MS2 minigene. An RNA-affinity-purified spliceosomal complex is immunoblotted with the indicated antibodies. U2AF65 and U2AF35 bind to the PPT and the $3^{\prime}$ ss, respectively. U1-70 K is a component of U1 snRNP that binds to the $5^{\prime}$ ss. A $\beta$-globin-MS2 pre-mRNA substrate is employed as a control. ${ }^{31} \mathrm{NE}$, nuclear extract (5\%). (c) ' $T$ ' (double underlined) is substituted for wild-type 'A' at position Int-9 to make an optimized PPT (Opt) carrying an uninterrupted stretch of 10 pyrimidines. A mutant ' $A$ ' nucleotide (shown in red) is serially introduced at positions Int-11: Int-3. PCR with reverse transcription of the minigenes introduced into HeLa cells are shown. The splicing consequences predicted by IntSplice, the Shapiro-Senapathy score-based model, and the MaxEntScan-based model are indicated. Incorrectly predicted consequences are marked by an asterisk. 'A', aberrant splicing; ' - ', normal splicing. (d) A spliceosome complex is purified and immunoblotted as in $\mathbf{b}$.

skipping (Figure 4c). MaxEntScan-based model may make the specificity high at the cost of lowering the sensitivity. As we have incorporated both the Shapiro-Senapathy score and MaxEntScan scores in our 110 parameters, we expected that the sensitivity and the specificity of IntSplice were superior to those of Shapiro-Senapathy score-based and MaxEntScan-based models. The better specificity of MaxEntScan compared with IntSplice is possibly accounted for because of the difference in the positions of the Int-SNVs used to produce their respective models: IntSplice was trained with Int-SNVs up to position Int-50, whereas MaxEntScan covered up to position Int-20. Alternatively, the MaxEntScan scores were underestimated among the 110 parameters in the SVM modeling of IntSplice for the sake of an improved sensitivity. Another possibility is that the higher specificity of MaxEntScan was lowered by the lower specificities of the other parameters used in the IntSplice modeling. We hope that our web service program, IntSplice, will reveal yet unidentified splicing mutations at positions Int-50:Int-3, and unveil aberrant splicing in human diseases.

\section{CONFLICT OF INTEREST}

The authors declare no conflict of interest.

\section{ACKNOWLEDGEMENTS}

The studies were supported by Grants-in-Aid from the MEXT and MHLW of Japan for AM and KO, and by NINDS Grant NS6277 for AGE.

1 Black, D. L. Mechanisms of alternative pre-messenger RNA splicing. Annu. Rev. Biochem. 72, 291-336 (2003).

2 Jurica, M. S. \& Moore, M. J. Pre-mRNA splicing: awash in a sea of proteins. Mol. Cell. 12, 5-14 (2003).

3 Reed, R. Initial splice-site recognition and pairing during pre-mRNA splicing. Curr. Opin. Genet. Dev. 6, 215-220 (1996). 
4 Gooding, C., Edge, C., Lorenz, M., Coelho, M. B., Winters, M., Kaminski, C. F. et al. MBNL1 and PTB cooperate to repress splicing of Tpm 1 exon 3. Nucleic Acids Res. 41, 4765-4782 (2013).

5 Krawczak, M., Thomas, N. S., Hundrieser, B., Mort, M., Wittig, M., Hampe, J. et al. Single base-pair substitutions in exon-intron junctions of human genes: nature, distribution, and consequences for mRNA splicing. Hum. Mutat. 28, 150-158 (2007).

6 Sterne-Weiler, T., Howard, J., Mort, M., Cooper, D. N. \& Sanford, J. R. Loss of exon identity is a common mechanism of human inherited disease. Genome Res. 21, 1563-1571 (2011).

7 Cartegni, L., Wang, J., Zhu, Z., Zhang, M. Q. \& Krainer, A. R. ESEfinder: a web resource to identify exonic splicing enhancers. Nucleic Acids Res. 31, 3568-3571 (2003).

8 Goren, A., Ram, O., Amit, M., Keren, H., Lev-Maor, G., Vig, I. et al. Comparative analysis identifies exonic splicing regulatory sequences-the complex definition of enhancers and silencers. Mol. Cell 22, 769-781 (2006).

9 Wang, Z., Rolish, M. E., Yeo, G., Tung, V., Mawson, M. \& Burge, C. B. Systematic identification and analysis of exonic splicing silencers. Cell 119, 831-845 (2004).

10 Zhang, Z. \& Krainer, A. R. Involvement of SR proteins in mRNA surveillance. Mol. Cell 16, 597-607 (2004).

11 Zhang, X. H., Kangsamaksin, T., Chao, M. S., Banerjee, J. K. \& Chasin, L. A. Exon inclusion is dependent on predictable exonic splicing enhancers. Mol. Cell. Biol. 25, 7323-7332 (2005).

12 Fairbrother, W. G., Yeh, R. F., Sharp, P. A. \& Burge, C. B. Predictive identification of exonic splicing enhancers in human genes. Science 297, 1007-1013 (2002).

13 Desmet, F. O., Hamroun, D., Lalande, M., Collod-Beroud, G., Claustres, M. \& Beroud, C. Human Splicing Finder: an online bioinformatics tool to predict splicing signals. Nucleic Acids Res. 37, e67 (2009).

14 Piva, F., Giulietti, M., Nocchi, L. \& Principato, G. SpliceAid: a database of experimental RNA target motifs bound by splicing proteins in humans. Bioinformatics 25, 1211-1213 (2009).

15 Piva, F., Giulietti, M., Burini, A. B. \& Principato, G. SpliceAid 2: a database of human splicing factors expression data and RNA target motifs. Hum. Mutat. 33, 81-85 (2012).

16 Divina, P., Kvitkovicova, A., Buratti, E. \& Vorechovsky, I. Ab initio prediction of mutation-induced cryptic splice-site activation and exon skipping.. Eur. J. Hum. Genet. 17, 759-765 (2009)

17 Lim, K. H., Ferraris, L., Filloux, M. E., Raphael, B. J. \& Fairbrother, W. G. Using positional distribution to identify splicing elements and predict pre-mRNA processing defects in human genes. Proc. Natl. Acad. Sci. USA 108, 11093-11098 (2011).

18 Chang, T. H., Huang, H. Y., Hsu, J. B., Weng, S. L., Horng, J. T. \& Huang, H. D. An enhanced computational platform for investigating the roles of regulatory RNA and for identifying functional RNA motifs. BMC Bioinformatics 14 (), S4 (2013).

19 Shapiro, M. B. \& Senapathy, P. RNA splice junctions of different classes of eukaryotes: sequence statistics and functional implications in gene expression. Nucleic Acids Res. 15, 7155-7174 (1987)

20 Yeo, G. \& Burge, C. B. Maximum entropy modeling of short sequence motifs with applications to RNA splicing signals. J. Comput. Biol. 11, 377-394 (2004).

21 Gao, K., Masuda, A., Matsuura, T. \& Ohno, K. Human branch point consensus sequence is yUnAy. Nucleic Acids Res. 36, 2257-2267 (2008).

22 Corvelo, A., Hallegger, M., Smith, C. W. \& Eyras, E. Genome-wide association between branch point properties and alternative splicing. PLoS Comput. Biol. 6, e1001016 (2010).

23 Taggart, A. J., DeSimone, A. M., Shih, J. S., Filloux, M. E. \& Fairbrother, W. G. Large-scale mapping of branchpoints in human pre-mRNA transcripts in vivo. Nat. Struct. Mol. Biol. 19, 719-721 (2012).
24 Bitton, D. A., Rallis, C., Jeffares, D. C., Smith, G. C., Chen, Y. Y., Codlin, S. et al. LaSSO, a strategy for genome-wide mapping of intronic lariats and branch points using RNA-seq. Genome Res. 24, 1169-1179 (2014).

$25 \mathrm{Fu}$, Y., Masuda, A., Ito, M., Shinmi, J. \& Ohno, K. AG-dependent 3'-splice sites are predisposed to aberrant splicing due to a mutation at the first nucleotide of an exon. Nucleic Acids Res. 39, 4396-4404 (2011).

26 Wang, E. T., Sandberg, R., Luo, S., Khrebtukova, I., Zhang, L., Mayr, C. et al. Alternative isoform regulation in human tissue transcriptomes. Nature 456, 470-476 (2008).

27 Pan, Q., Shai, O., Lee, L. J., Frey, B. J. \& Blencowe, B. J. Deep surveying of alternative splicing complexity in the human transcriptome by high-throughput sequencing. Nat. Genet. 40, 1413-1415 (2008).

28 Trapnell, C., Pachter, L. \& Salzberg, S. L. TopHat: discovering splice junctions with RNA-Seq. Bioinformatics 25, 1105-1111 (2009).

29 Katz, Y., Wang, E. T., Airoldi, E. M. \& Burge, C. B. Analysis and design of RNA sequencing experiments for identifying isoform regulation. Nat. Methods 7, 1009-1015 (2010)

30 Chang, C. C. \& Lin, C. J. LIBSVM: A Library for Support Vector Machines. ACM T Intel Syst Tec. 2, Article 27 (2011).

31 Rahman, M. A., Masuda, A., Ohe, K., Ito, M., Hutchinson, D. O., Mayeda, A. et al. HnRNP L and hnRNP LL antagonistically modulate PTB-mediated splicing suppression of CHRNA1 pre-mRNA. Sci. Rep. 3, 2931 (2013).

32 Ohno, K., Anlar, B., Ozdirim, E., Brengman, J. M., DeBleecker, J. L. \& Engel, A. G. Myasthenic syndromes in Turkish kinships due to mutations in the acetylcholine receptor. Ann. Neurol. 44, 234-241 (1998).

33 Das, R., Zhou, Z. \& Reed, R. Functional association of U2 snRNP with the ATP-independent spliceosomal complex E. Mol. Cell 5, 779-787 (2000).

34 Zuker, M. Mfold web server for nucleic acid folding and hybridization prediction. Nucleic Acids Res. 31, 3406-3415 (2003).

35 Gahura, O., Hammann, C., Valentova, A., Puta, F. \& Folk, P. Secondary structure is required for $3^{\prime}$ splice site recognition in yeast. Nucleic Acids Res. 39, 9759-9767 (2011).

36 Plass, M., Codony-Servat, C., Ferreira, P. G., Vilardell, J. \& Eyras, E. RNA secondary structure mediates alternative 3'ss selection in Saccharomyces cerevisiae. RNA 18, 1103-1115 (2012)

37 Pervouchine, D. D., Khrameeva, E. E., Pichugina, M. Y., Nikolaienko, O. V., Gelfand, M. S., Rubtsov, P. M. et al. Evidence for widespread association of mammalian splicing and conserved long-range RNA structures. RNA 18, 1-15 (2012).

38 Ohno, K., Engel, A. G., Shen, X. M., Selcen, D., Brengman, J., Harper, C. M. et al. Rapsyn mutations in humans cause endplate acetylcholine-receptor deficiency and myasthenic syndrome. Am J Hum Genet. 70, 875-885 (2002).

39 Milone, M., Shen, X. M., Selcen, D., Ohno, K., Brengman, J., lannaccone, S. T. et al. Myasthenic syndrome due to defects in rapsyn: clinical and molecular findings in 39 patients. Neurology 73, 228-235 (2009).

40 Kornblihtt, A. R., Schor, I. E., Allo, M., Dujardin, G., Petrillo, E. \& Munoz, M. J. Alternative splicing: a pivotal step between eukaryotic transcription and translation. Nat. Rev. Mol. Cell Biol. 14, 153-165 (2013).

41 Giulietti, M., Piva, F., D'Antonio, M., D'Onorio De, M. P., Paoletti, D., Castrignano, T. et al. SpliceAid-F: a database of human splicing factors and their RNA-binding sites. Nucleic Acids Res. 41, D125-D131 (2013).

42 Rahman, M. A., Nasrin, F., Masuda, A. \& Ohno, K. Decoding abnormal splicing code in human diseases. J. Invest. Genomics 2, 00016 (2015).

43 Rieder, L. E. \& Reenan, R. A. The intricate relationship between RNA structure, editing, and splicing. Semin. Cell Dev. Biol. 23, 281-288 (2012).

Supplementary Information accompanies the paper on Journal of Human Genetics website (http://www.nature.com/jhg) 